\title{
Starspot activity and rotation of the planet-hosting star Kepler-17
}

\author{
A. S. Bonomo ${ }^{1}$ and A. F. Lanza ${ }^{2}$
}

\author{
1 INAF - Osservatorio Astrofisico di Torino, via Osservatorio, 20, 10025 Pino Torinese, Italy \\ e-mail: bonomo@oato.inaf.it \\ 2 INAF - Osservatorio Astrofisico di Catania, via S. Sofia, 78, 95123 Catania, Italy \\ e-mail: nuccio.lanza@oact.inaf.it
}

Received 12 July 2012 / Accepted 20 September 2012

\begin{abstract}
Context. Kepler-17 is a G2V sun-like star accompanied by a transiting planet with a mass of $\approx 2.5$ Jupiter masses and an orbital period of $1.486 \mathrm{~d}$, recently discovered by the Kepler space telescope. This star is highly interesting as a young solar analogue. It is also a good candidate for a test of the tidal theories for solar-like stars.

Aims. We used about 500 days of high-precision, high-duty-cycle optical photometry collected by Kepler to study the rotation of the star and the evolution of its photospheric active regions.

Methods. We applied a maximum-entropy light curve inversion technique to model the flux rotational modulation induced by active regions that consist of dark spots and bright solar-like faculae with a fixed area ratio. Their configuration was varied after a fixed time interval to take their evolution into account. Active regions were used as tracers to study stellar differential rotation, and planetary occultations were used to constrain the latitude of some spots.

Results. Our modelling approach reproduces the light variations of Kepler-17 with a standard deviation of the residuals comparable with the precision of Kepler photometry. We find several active longitudes where individual active regions appear, evolve, and decay with lifetimes comparable to those observed in the Sun, although the star has a spotted area $\approx 10-15$ times larger than the Sun at the maximum of the 11-yr cycle. Kepler-17 shows a solar-like latitudinal differential rotation, but the fast spot evolution prevents a precise determination of its amplitude. Moveover, the star shows a cyclic variation of the starspot area with a period of $47.1 \pm 4.5 \mathrm{~d}$, particularly evident during the last 200 days of the observations, similar to the solar Rieger cycles. Possible effects of the close-in massive planet on stellar photospheric activity cannot be excluded, but require a long-term monitoring to be unambiguously detected.
\end{abstract}

ABSTRACT

Key words. stars: late-type - stars: activity - stars: rotation - planetary systems - stars: individual: Kepler-17

\section{Introduction}

The Kepler space mission has recently announced the discovery of the hot Jupiter Kepler-17b. It orbits a G2V star with $K_{p}{ }^{1}=14.14$ in $P=1.486$ d (Désert et al. 2011, hereafter D11). Thanks to additional spectra acquired with the SOPHIE spectrograph at the Observatoire de Haute Provence, Bonomo et al. (2012; hereafter B12) slightly refined the characterization of the Kepler-17 planetary system. In particular, they found the host star to be slightly hotter and younger than D11, with an effective temperature of $5781 \pm 85 \mathrm{~K}$ and an age $<1.8 \mathrm{Gyr}$.

The hosting star Kepler-17 is remarkably active, as shown by the out-of-transit variations in the Kepler light curve with a peak-to-peak amplitude of $\approx 4 \%$. They are produced by the rotational modulation of active regions, which consist of starspots and faculae, on the stellar disc. By means of a periodogram analysis of the light curve, D11 estimated the stellar rotation period to be $P_{\text {rot }}=11.89 \pm 0.15 \mathrm{~d}$. Intriguingly, this value of $P_{\text {rot }}$ is an integer multiple of the orbital period, specifically eight times. This could be just a coincidence or the result of a star-planet interaction, as pointed out by D11.

In addition, the planet occults starspots during transits, giving rise to the typical bumps observed in the bottom of the transit profiles (e.g., Silva-Valio et al. 2010; Silva-Valio \& Lanza 2011). The integer ratio between $P_{\text {rot }}$ and $P$ allowed D11 to see a "stroboscopic" effect with the short-cadence Kepler data: the spots are "mapped" by the planet each $45^{\circ}$ in longitude. The study of these spot-crossing events reveals that the planet's orbit

\footnotetext{
1 Kepler magnitude (cf. Sect. 2).
}

is prograde and the projected spin-orbit angle is smaller than $10^{\circ}-15^{\circ}$ (see D11 for more details).

Kepler-17 is therefore an excellent candidate to study the magnetic activity in a solar-like star younger than the Sun, through the modelling of its out-of-transit flux variations. A similar analysis has been performed for several CoRoT planethosting stars. One of them is CoRoT-2, an active G7V star with a rotation period of $4.5 \mathrm{~d}$ and an age younger than $0.5 \mathrm{Gyr}$ (Alonso et al. 2008). The spot-modelling of its light curve revealed two active longitudes and a cyclic oscillation of the total spotted area with a period of $28.3 \pm 4.3 \mathrm{~d}$, similarly to the solar Rieger cycles (Lanza et al. 2009a). Investigating the magnetic activity of $\mathrm{G}$ dwarfs with different ages would in principle allow one to study the Sun in time. Recently, Frasca et al. (2011) and Fröhlich et al. (2012) have applied a spot-modelling to Kepler photometric time series to study three young G- and K-type dwarf stars whose ages range between $\approx 50$ and $\approx 200 \mathrm{Myr}$, deriving information on their spot evolution and surface differential rotation.

Owing to the faintness and low projected rotation velocity of Kepler-17 $\left(v \sin i \approx 5 \mathrm{~km} \mathrm{~s}^{-1}\right.$ ), an investigation of its magnetic activity through Doppler imaging is not feasible. Therefore, modelling the stellar variability and studying the distortions of the transit profile when the planet occults starspots are the only techniques that allow us to derive active longitudes where spots preferentially form, determine the lifetime of active regions and activity complexes, estimate a minimum amplitude for the stellar differential rotation, and possibly discover short-term and/or long-term activity cycles, depending on the length of the time series. 


\section{Observations}

The Kepler space telescope has an aperture of $95 \mathrm{~cm}$ and is designed to yield nearly continuous, high-precision photometry in the passband $423-897 \mathrm{~nm}$ for $\approx 150000$ stars in a fixed field of view to search for planetary transits (see, e.g., Borucki et al. 2010; Koch et al. 2010). It orbits the Sun on an Earth-trailing orbit and, to keep the solar arrays pointed towards the Sun, a re-orientation of the spacecraft is required every $\approx 90$ days, a time interval called "a quarter" in the Kepler jargon. The reorientation of the telescope produces an offset in the photon counting for a given star because its photometric mask is redefined on a different CCD in the focal plane.

The light curve of Kepler-17, publicly available at the MAST archive $^{2}$, covers more than fifteen months of photometric measurements, from 2009 May 13 to 2010 August 23. Observations are distributed along the six quarters Q1-Q6. The raw data with the long-cadence temporal sampling, i.e. one point each $29.42 \mathrm{~min}$, were used for our work. Short-cadence data (one point per minute) available for the last three quarters (Q4-Q6) are not particularly useful for our purpose because we are interested in modelling the out-of-transit variations on the timescale of stellar rotation or longer.

Because of the flux offsets between adjacent quarters and the long-term instrumental trends within each quarter (see Jenkins et al. 2010a, and Fig. 1 in D11 for our specific case), the light curves corresponding to different quarters were separately treated as follows. First, planetary transits were removed from each quarter. The flux contamination due to starfield crowding, as estimated by the Kepler team, was subtracted from the median value of the flux. Steep variations after the safe modes (Jenkins et al. 2010b) were removed and long-term trends of clear instrumental origin were corrected by fitting a parabola. Lastly, the flux of each quarter was normalized to its median value then nearly matched the endpoints of adjacent quarters. The final light curve obtained by combining the six quarters contains 20924 data points and is shown in Sect. 5.1 (see Fig. 2). The median of the errors of the single photometric measurements is $2.18 \times 10^{-4}$ in relative flux units.

\section{Spot modelling of wide-band light curves}

Reconstructing the surface brightness distribution from the rotational modulation of the stellar flux is an ill-posed problem, because the variation of the flux vs. rotational phase contains information only on the distribution of the brightness inhomogeneities vs. longitude. The integration over the stellar disc effectively cancels any latitudinal information, particularly when the inclination of the rotation axis along the line of sight is close to $90^{\circ}$, as in the present case (see Sect. 4 and Lanza et al. 2009a). Therefore, we need to include a priori information in the light curve inversion process to obtain a unique and stable map. This is done by computing a maximum entropy (hereinafter ME) map, which has been proven to best reproduce active region distribution and area variations for the Sun (cf. Lanza et al. 2007). For a different modelling approach, based on discrete starspots, see, e.g., Mosser et al. (2009). A comparison with other modelling approaches is given in, e.g., Fröhlich et al. (2009); Huber et al. (2010); Silva-Valio \& Lanza (2011).

In the present model, the stellar surface is subdivided into elements, i.e., into 200 squares of side $18^{\circ}$, with each element containing unperturbed photosphere, dark spots, and facular areas. The fraction of the $k$ th element covered by dark spots is

\footnotetext{
${ }^{2}$ http://archive.stsci.edu/kepler/data_search/search.php
}

indicated by its filling factor $f_{k}$, the fractional area of the faculae is $Q f_{k}$, and the fractional area of the unperturbed photosphere is $1-(Q+1) f_{k}$. The contribution to the stellar flux coming from the $k$ th surface element at time $t_{j}$, where $j=1, \ldots, N$ is an index numbering the $N$ points along the light curve, is given by

$$
\begin{aligned}
\Delta F_{k j}= & I_{0}\left(\mu_{k j}\right)\left\{1-(Q+1) f_{k}+c_{\mathrm{s}} f_{k}\right. \\
& \left.+Q f_{k}\left[1+c_{\mathrm{f}}\left(1-\mu_{k j}\right)\right]\right\} A_{k} \mu_{k j} w\left(\mu_{k j}\right),
\end{aligned}
$$

where $I_{0}$ is the specific intensity in the continuum of the unperturbed photosphere at the isophotal wavelength of the observations, $c_{\mathrm{s}}$ and $c_{\mathrm{f}}$ are the spot and facular contrasts, respectively (cf. Lanza et al. 2004), $A_{k}$ is the area of the $k$ th surface element,

$w\left(\mu_{k j}\right)= \begin{cases}1 & \text { if } \mu_{k j} \geq 0 \\ 0 & \text { if } \mu_{k j}<0\end{cases}$

is its visibility, and

$\mu_{k j} \equiv \cos \psi_{k j}=\sin i \sin \theta_{k} \cos \left[\ell_{k}+\Omega\left(t_{j}-t_{0}\right)\right]+\cos i \cos \theta_{k}$,

is the cosine of the angle $\psi_{k j}$ between the normal to the surface element and the direction of the observer, with $i$ being the inclination of the stellar rotation axis to the line of sight, $\theta_{k}$ the colatitude and $\ell_{k}$ the longitude of the $k$ th surface element, $\Omega$ the angular velocity of rotation of the $\operatorname{star}\left(\Omega \equiv 2 \pi / P_{\text {rot }}\right)$, and $t_{0}$ the initial time. The specific intensity in the continuum varies according to a quadratic limb-darkening law, as adopted by Lanza et al. (2003) for the Sun, viz. $I_{0} \propto a_{\mathrm{p}}+b_{\mathrm{p}} \mu+c_{\mathrm{p}} \mu^{2}$. The stellar flux computed at time $t_{j}$ is then: $F\left(t_{j}\right)=\sum_{k} \Delta F_{k j}$. To warrant a relative precision of about $10^{-5}$ in the computation of the flux $F$, each surface element is further subdivided into elements of $1^{\circ} \times 1^{\circ}$ and their contributions, calculated according to Eq. (1), are summed up at each given time to compute the contribution of the $18^{\circ} \times 18^{\circ}$ surface element to which they belong.

We fitted the light curve by changing the value of the spotfilling factor $f$ over the surface of the star while $Q$ was held constant. Even fixing the rotation period, the inclination, and the spot and facular contrasts (see Lanza et al. 2007, for details), the model has 200 free parameters and suffers from non-uniqueness and instability. To find a unique and stable spot map, we applied ME regularization, as described in Lanza et al. (2007), by minimizing a functional $Z$, which is a linear combination of the $\chi^{2}$ and the entropy functional $S$; i.e.,

$Z=\chi^{2}(\boldsymbol{f})-\lambda S(\boldsymbol{f})$,

where $f$ is the vector of the filling factors of the surface elements, $\lambda>0$ a Lagrangian multiplier determining the trade-off between light curve fitting and regularization, and the expression of $S$ is

$S=-\sum_{k} w_{k}\left[f_{k} \log \frac{f_{k}}{m}+\left(1-f_{k}\right) \log \frac{1-f_{\mathrm{k}}}{1-m}\right]$,

where $w_{k}$ is the relative area of the $k$ th surface element (total surface area of the star $=1$ ) and $m$ the default spot-covering factor that fixes the limiting values for $f_{k}$ as: $m<f_{k}<(1-m)$. In our modelling we adopted $m=10^{-6}$ (cf. Lanza et al. 1998). The entropy functional $S$ is constructed in such a way that it attains its maximum value when the star is virtually immaculate $\left(f_{k}=m\right.$ for every $k$ ). Therefore, by increasing the Lagrangian multiplier $\lambda$, the weight of $S$ in the model is increased and the 
area of the spots is progressively reduced. This gives rise to systematically negative residuals between the observations and the best-fit model when $\lambda>0$.

The optimal value of $\lambda$ depends on the information content of the light curve, which in turn depends on the ratio of the amplitude of its rotational modulation to the average standard deviation of its points. In the case of Kepler-17, the amplitude of the rotational modulation is $\approx 0.044$, while the nominal standard deviation of the points is $\approx 2.18 \times 10^{-4}$ in relative flux units (see Sect. 2), giving a signal-to-noise ratio of $\approx 200$.

To fix the optimal value of the Lagrangian multiplier $\lambda$, we compared the modulus of the mean of the residuals of the regularized best fit $\left|\mu_{\text {reg }}\right|$ with the standard error of the residuals themselves, i.e., $\epsilon_{0} \equiv \sigma_{0} / \sqrt{N}$, where $\sigma_{0}$ is the standard deviation of the residuals of the unregularized best fit and $N$ the number of points in each fitted subset of the light curve having a duration $\Delta t_{\mathrm{f}}$ (see below). We iterated until $\left|\mu_{\text {reg }}\right| \simeq \epsilon_{0}$ (cf. the case of CoRoT-2 in Lanza et al. 2009a).

For the Sun, by assuming a fixed distribution of the filling factor, it is possible to obtain a good fit of the irradiance changes only for a limited time interval $\Delta t_{\mathrm{f}}$, not exceeding 14 days, which is the lifetime of the largest sunspot groups dominating the irradiance variation (Lanza et al. 2003). For other active stars, the value of $\Delta t_{\mathrm{f}}$ must be determined from the observations themselves, looking for the maximum data extension that allows us a good fit with the applied model (see Sect. 4).

The optimal values of the spot and facular contrasts and of the facular-to-spotted area ratio $Q$ in stellar active regions are unknown a priori. In our model the facular contrast $c_{\mathrm{f}}$ and the parameter $Q$ enter as the product $c_{\mathrm{f}} Q$, so we can fix $c_{\mathrm{f}}$ and vary $Q$, estimating its best value by minimizing the $\chi^{2}$ of the model, as shown in Sect. 4. Since the number of free parameters of the ME model is large, we used the model of Lanza et al. (2003) to fix the value of $Q$. It fits the light curve by assuming only three active regions to model the rotational modulation of the flux plus a uniformly distributed background to account for the variations of the mean light level. This procedure is the same as adopted for, e.g., CoRoT-2 and CoRoT-4 to fix the value of $Q$ (cf. Lanza et al. 2009a,b).

We assumed an inclination of the rotation axis of Kepler-17 of $i=87.2^{\circ}$ (see Sect. 4). Since the information on spot latitudes that can be extracted from the rotational modulation of the flux for such a high inclination is negligible, the ME regularization virtually puts all spots at the sub-observer latitude (i.e., $90^{\circ}-i \approx 3^{\circ}$ ) to minimize their area and maximize the entropy. Therefore, we are limited to mapping only the distribution of the active regions vs. longitude, which can be achieved with a resolution of at least $\approx 40^{\circ}-50^{\circ}$ (cf. Lanza et al. 2007, 2009a). Our ignorance of the true facular contribution to the light modulation may lead to systematic errors in the active region longitudes derived by our model, as discussed by Lanza et al. (2007) for the Sun.

\section{Model parameters}

The basic stellar parameters are taken from D11 and B12 and are listed in Table 1. The limb-darkening parameters in the Kepler bandpass were derived from the model of the transit as discussed in D11.

The rotation period for our spot modelling was initially fixed at exactly eight orbital periods of the planet, following D11, who found that there were spots occulted by the planet that reappeared at the same phase after that time interval, i.e., $11.89 \mathrm{~d}$.
Table 1. Parameters adopted for the modelling of the light curve of Kepler-17.

\begin{tabular}{lcc}
\hline \hline Parameter & Value & Ref. \\
\hline Star mass $\left(M_{\odot}\right)$ & 1.16 & B12 \\
Star radius $\left(R_{\odot}\right)$ & 1.05 & B12 \\
$T_{\text {eff }}(\mathrm{K})$ & 5780 & B12 \\
$\log g\left(\mathrm{~cm} \mathrm{~s}^{-2}\right)$ & 4.53 & B12 \\
$a_{\mathrm{p}}$ & 0.333 & BL12 \\
$b_{\mathrm{p}}$ & 0.929 & BL12 \\
$c_{\mathrm{p}}$ & -0.262 & BL12 \\
$P_{\text {rot }}$ (days $)$ & 12.01 & BL12 \\
$\epsilon_{\text {rot }}$ & $4.66 \times 10^{-5}$ & BL12 \\
Inclination $($ deg $)$ & 87.22 & D11 \\
$c_{\mathrm{s}}$ & 0.677 & L04 \\
$c_{\mathrm{f}}$ & 0.115 & L04 \\
$Q$ & 1.6 & BL12 \\
$\Delta t_{\mathrm{f}}$ (days) & 8.733 & BL12 \\
\hline
\end{tabular}

References. B12: Bonomo et al. (2012); D11: Désert et al. (2011); L04: Lanza et al. (2004); BL12: present study.

However, we found that the migration rates of the other spots revealed by the modelling of the out-of-transit photometry was minimized by assuming a slightly different rotation period, i.e., $12.01 \mathrm{~d}$, which we adopted for our modelling.

The polar flattening of the star owing to the centrifugal potential was computed in the Roche approximation with a rotation period of $12.01 \mathrm{~d}$. The relative difference between the equatorial and the polar radii is $\epsilon_{\mathrm{rot}}=4.66 \times 10^{-5}$, which induces a completely negligible relative flux variation of $\approx 2 \times 10^{-6}$ for a spot coverage of $\approx 4$ percent, as a consequence of the gravity darkening of the equatorial regions of the star.

The inclination of the stellar rotation axis is constrained by the observation of D11 that the light anomalies detected in successive transits are compatible with the same spots being repeatedly occulted as they move over the stellar disc owing to stellar rotation. This is possible only if the disc-projected trajectory of the spots remains inside the belt occulted by the planet. In other words, this is an indication that the sky-projected misalignment between the stellar spin and the orbital angular momentum is smaller than $\pm\left(10^{\circ}-15^{\circ}\right)$ (cf. D11 for details). Although the possibility that the stellar spin is not aligned with the orbital angular momentum cannot be excluded with certainty because only the sky-projected angle between the two vectors has been measured, we regard this occurrence as highly improbable and assume that the rotation axis is inclined with respect to the line of sight of the same angle as the orbit normal. Moreover, the stellar $v \sin i=4.7 \pm 1.0 \mathrm{~km} \mathrm{~s}^{-1}$ and the estimated stellar radius provide an inclination close to $90^{\circ}$ for the stellar rotation period (cf. D11).

The maximum time interval $\Delta t_{\mathrm{f}}$ that our model can accurately fit with a fixed distribution of active regions was determined by dividing the total interval, $T=497.785 \mathrm{~d}$, into $N_{\mathrm{f}}$ equal segments, i.e., $\Delta t_{\mathrm{f}}=T / N_{\mathrm{f}}$, and looking for the minimum value of $N_{\mathrm{f}}$ that allows us a good fit of the light curve, as measured by the $\chi^{2}$ statistics. We found that for $N_{\mathrm{f}}<57$ the quality of the best fit degrades appreciably with respect to higher values, owing to a substantial evolution of the pattern of surface brightness inhomogeneities. Therefore, we adopted $N_{\mathrm{f}}=57$, so that $\Delta t_{\mathrm{f}}=8.733 \mathrm{~d}$ is the maximum time interval to be fitted with a fixed distribution of surface active regions to estimate the best value of the parameter $Q$ (see below). 


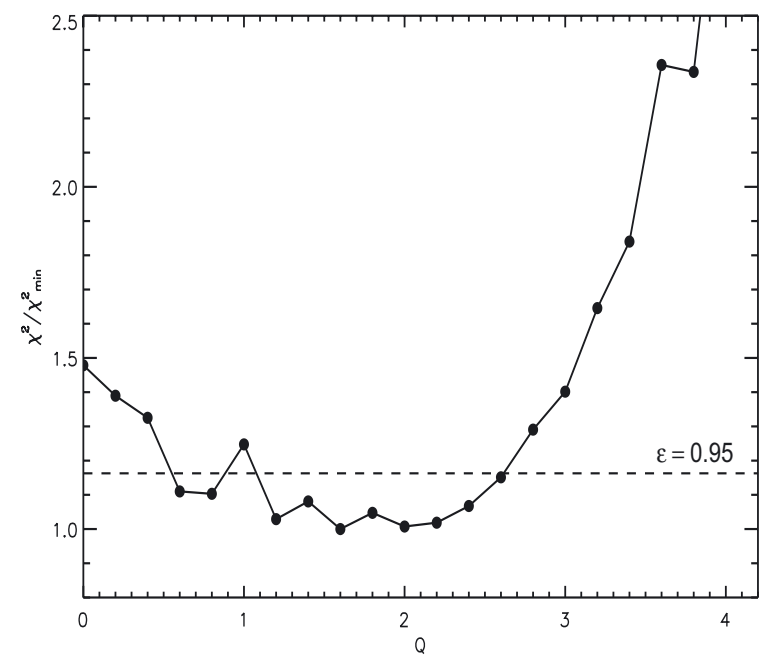

Fig. 1. Ratio of the $\chi^{2}$ of the composite best fit of the entire time series of Kepler- 17 to its minimum value vs. the parameter $Q$, i.e., the ratio of the area of the faculae to that of the cool spots in active regions. The horizontal dashed line indicates the 95 percent confidence level for $\chi^{2} / \chi_{\min }^{2}$, determining the interval of acceptable $Q$ values.

To evaluate the spot contrast, we adopted the same mean temperature difference as derived for sunspot groups from their bolometric contrast, i.e. $560 \mathrm{~K}$ (Chapman et al. 1994). The effective temperature of the unspotted photosphere is $5780 \pm 85 \mathrm{~K}$, i.e., very similar to that of the Sun (cf. B12). In other words, we assumed a spot effective temperature of $5220 \mathrm{~K}$, yielding a contrast $c_{\mathrm{s}}=0.677$ in the bolometric passband (cf. Lanza et al. 2007). A different spot contrast changes the absolute spot coverages, but significantly affects neither their longitudes nor their time evolution, as discussed in detail by Lanza et al. (2009a). Therefore, since the spot temperature is only estimated, we neglected the difference in the contrast $c_{\mathrm{s}}$ between the Kepler bandpass and the bolometric passband in our analysis.

In our model, the facular contrast is assumed to be solar-like with $c_{\mathrm{f}}=0.115$ (Lanza et al. 2004). The best value of the area ratio $Q$ between the faculae and the spots in the active regions was estimated by means of the three-spot model by Lanza et al. (2003, cf. Sect. 3). In Fig. 1, we plot the ratio $\chi^{2} / \chi_{\min }^{2}$ of the total $\chi^{2}$ of the composite best fit of the entire time series to its minimum value $\chi_{\min }^{2}$, versus $Q$, and indicate the 95 percent confidence level as derived from the F-statistics (e.g., Lampton et al. 1976). The choice of $\Delta t_{\mathrm{f}}=8.733 \mathrm{~d}$ allows us to fit the rotational modulation of the active regions for the longest time interval during which they remain stable, modelling both the flux increase due to the facular component when an active region is close to the limb and the flux decrease due to the dark spots when the same region transits across the central meridian of the disc. In this way, a measure of the relative facular and spot contributions can be obtained, leading to an estimate of $Q$. Figure 1 shows that the best value is $Q=1.6$, with an acceptable range extending from $\approx 0.6$ to $\approx 2.6$. Therefore, we adopted $Q=1.6$ for our modelling in Sect. 5. We comment on the value of $Q$ in more detail in Sect. 6.

\section{Results}

\subsection{Light curve models}

We applied the model of Sect. 3 to the out-of-transit light curve of Kepler-17, considering time intervals $\Delta t_{\mathrm{f}}=8.733 \mathrm{~d}$. The best fit without regularization $(\lambda=0)$ has a mean $\mu_{\text {res }}=3.845 \times 10^{-6}$ and a standard deviation of the residuals $\sigma_{0}=3.032 \times 10^{-4}$ in relative flux units. The Lagrangian multiplier $\lambda$ is iteratively adjusted until the mean of the residuals $\mu_{\text {res }}=-1.583 \times 10^{-5} \simeq$ $-\sigma_{0} / \sqrt{N}$, where $N=367$ is the mean number of points in each fitted light curve interval $\Delta t_{\mathrm{f}}$; the standard deviation of the residuals of the regularized best fit is $\sigma=3.311 \times 10^{-4}$.

The composite best fit to the entire light curve is shown in the upper panel of Fig. 2, while the residuals are plotted in the lower panel. The best fit is always very good, with a standard deviation of the residuals $\approx 1.52$ times the median of the errors of the photometric measurements as given by the Kepler pipeline. The distribution of the residuals is plotted in Fig. 3 and is well fitted by a Gaussian with a standard deviation of $3.056 \times 10^{-4}$ for absolute values of the residuals lower than $\approx 6 \times 10^{-4}$ in relative flux units. For residuals greater than $\approx 6 \times 10^{-4}$ there is a remarkable asymmetry in the distribution with an excess of positive residuals. A periodogram of the residuals shows a highly significant peak at a period of $1.505 \pm 0.005 \mathrm{~d}$, very close to the orbital period, and several lower peaks at periods between $\approx 1.2$ and $\approx 3 \mathrm{~d}$. Putting the residuals in phase with the orbital period, we see the occultation of the planet and a possible phase-dependence of the reflected light (see B12).

\subsection{Longitude distribution of active regions and stellar differential rotation}

The distribution of the spot-filling factor $f$ vs. the longitude and the time is plotted in Fig. 4. The longitude zero corresponds to the point intercepted on the stellar photosphere by the line of sight to the centre of the star at BJD 2454964.5109, i.e., the subobserver point at the initial epoch. The reference frame rotates with the star with a fixed period of $12.01 \mathrm{~d}$ and the longitude increases in the same direction as the stellar rotation and the orbital motion of the planet. This is consistent with the reference frames adopted in our previous studies (e.g., Lanza et al. 2009a,b), but does not allow a direct comparison of the mapped active regions with the dips in the light curve.

Our map shows that the individual starspots evolve with timescales of tens of days, which makes it difficult to trace the evolution and migration of the active regions in an unambiguous way. Nevertheless, two main active longitudes, where individual spots form and evolve, can be identified with confidence and appear to rotate on the whole with the rotation period of our reference frame. The migration rate of individual spots or groups of spots within each longitude is variable, which suggests that individual spots are forming at different latitudes on a differentially rotating star or, alternatively, there are several spots at close longitudes that are evolving to mimic spot migration. Unfortunately, there is no information on the starspot latitude from our mapping technique and even the hemisphere cannot be determined because the inclination is close to $90^{\circ}$ (cf. Sect. 3). Nevertheless, some additional information can be extracted in this particular case from the occultation of the spots by the planet during transits. Specifically, we can exploit the results of D11 to constrain the latitude of some of the starspot trails that are seen in Fig. 4.

Désert et al. (2011) identified five starspots that are repeatedly occulted every eight transits and labelled them A, B, C, D, and $\mathrm{E}$ (cf. their Figs. 11 and 12). The transit profile distortions associated with spot $\mathrm{C}$ are barely visible in their Fig. 11, thus spot $\mathrm{C}$ is certainly significantly smaller than the other four spots. We plot in Fig. 4 the migration of the starspots detected by D11. The initial epoch $E(0)$ of D11 is equal to the epoch of the first mid transit as reported in their Table 3 (Désert, priv. comm.) so 


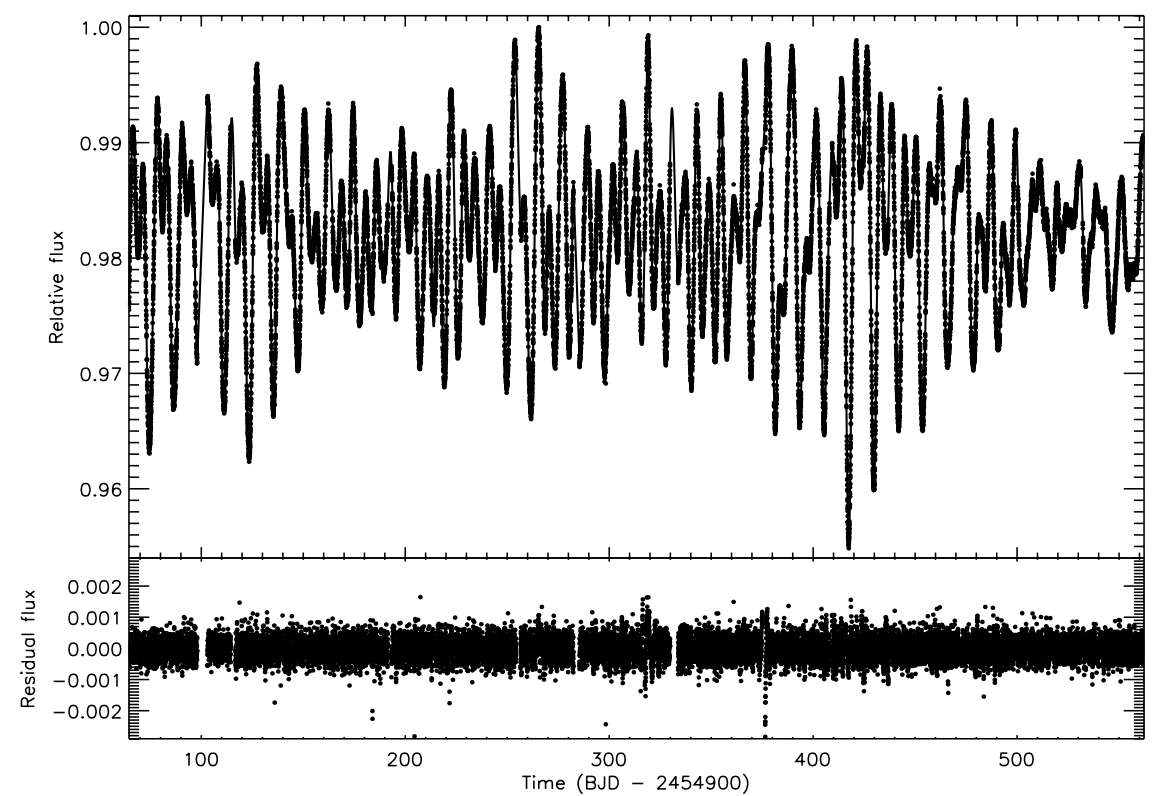

Fig. 2. Upper panel: the ME-regularized composite best fit to the out-of-transit light curve of Kepler-17 obtained for $Q=1.6$. The flux is in relative units, i.e., normalized to the maximum observed flux along the light curve. Lower panel: the residuals from the composite best fit versus the time.

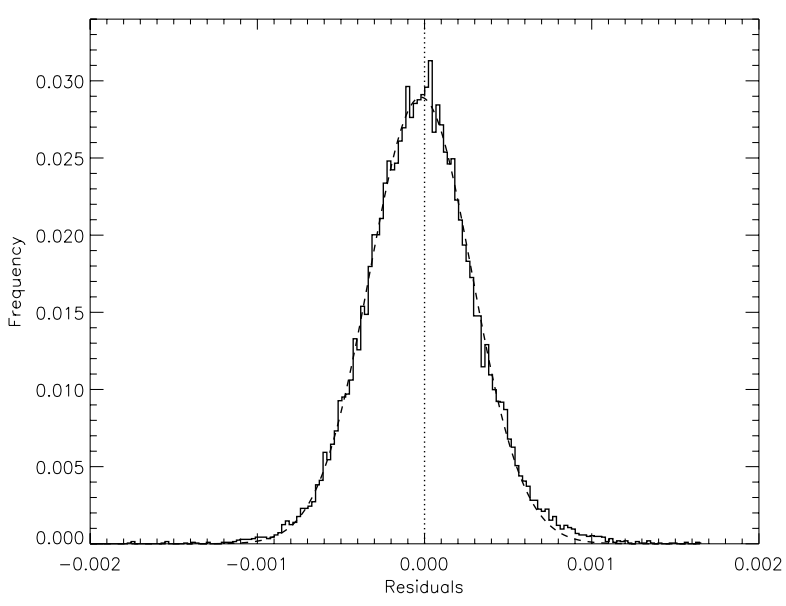

Fig. 3. Distribution of the residuals to the composite ME-regularized best fit shown in Fig. 2. The dashed line is a Gaussian fit to the residual distribution, while the vertical dotted line marks the zero value of the residuals.

we can easily convert their phases of maximum starspot visibility into longitudes in our reference frame. We find a good association between their starspots $\mathrm{A}, \mathrm{B}, \mathrm{D}$, and $\mathrm{E}$ and some trails of spots found with our approach. For starspot $C$ we find almost no coincidence, as expected owing to its smaller spotted area. The resolution of the transit-mapping method is of the order of some degrees (cf., e.g., Silva-Valio et al. 2010; Silva-Valio \& Lanza 2011, and references therein) while that of our mapping based on the out-of-transit light curve is approximately $40^{\circ}-50^{\circ}$. Therefore, we are not able to resolve the occulted spots with the same detail as D11, especially when there are several small spots close in longitude. Note that a detailed comparison of the spot maps based on the in-transit and out-of-transit photometry is outside the scope of the present work because it requires a detailed modelling of the transit profile distortions induced by occulted starspots (see Silva-Valio \& Lanza 2011, for such a detailed comparison for CoRoT-2). Here we limit ourselves to exploiting the preliminary modelling of D11 to find that our trails of spots with a positive migration rate, i.e., rotating faster than our reference frame, are located inside the belt that is occulted by the planet. A faster migration is particularly evident in the case of our spot trails associated with their spots B and D, at least for a significant part of the considered time interval. On the other hand, the intrinsic evolution of the pattern makes the determination of the migration rates of our spots associated with their spots $\mathrm{A}$ and $\mathrm{E}$ less certain, although a positive migration is suggested. On the whole, these results indicate that the occulted spots located at low latitudes are rotating faster than the overall pattern of the active longitudes, thus giving evidence of a solar-like differential rotation in Kepler-17, i.e., with the equator rotating faster than the poles.

Finally, we note that the time resolution of our spot models is not adequate to look for a possible modulation of the stellar activity with the orbital period of its close-in massive planet, as suggested by Shkolnik et al. (2008) or Lanza (2011). Given the short orbital period of the planet, a different approach should be used to search for signatures of a possible star-planet interaction using short-cadence data, as for CoRoT-2 (cf., e.g., Pagano et al. 2009).

\subsection{Variation of the spotted area}

The lifetimes of the active longitudes traced in Fig. 4 range from $\approx 75$ to $\approx 330$ days and possibly longer, given the limited time interval covered by our observations. Individual active regions have a lifetime of about a few tens of days. This is similar to what we observe in the Sun where complexes of activity consisting of several active regions, forming approximately around the same longitude, have lifetimes up to 5-6 months. The duration of the light anomalies observed during the transits reaches to $\approx 0.02$ in phase units, indicating a size of the largest occulted active regions of at least $\approx 40^{\circ}$ in longitude, i.e., $3-4$ times that of the largest sunspot groups.

The variation of the total starspot area vs. the time is plotted in Fig. 5. The error bars have a semi-amplitude of three standard deviations as derived from the uncertainty of the photometry, but systematic errors associated with the assumptions of the data reduction and the model are not included. Specifically, the variations on time scales longer than the duration of one quarter, i.e., 90-100 days, cannot be reconstructed from Kepler photometry because of the flux jumps from one quarter to the next (see Sect. 2). Therefore, we are limited to study the variations 

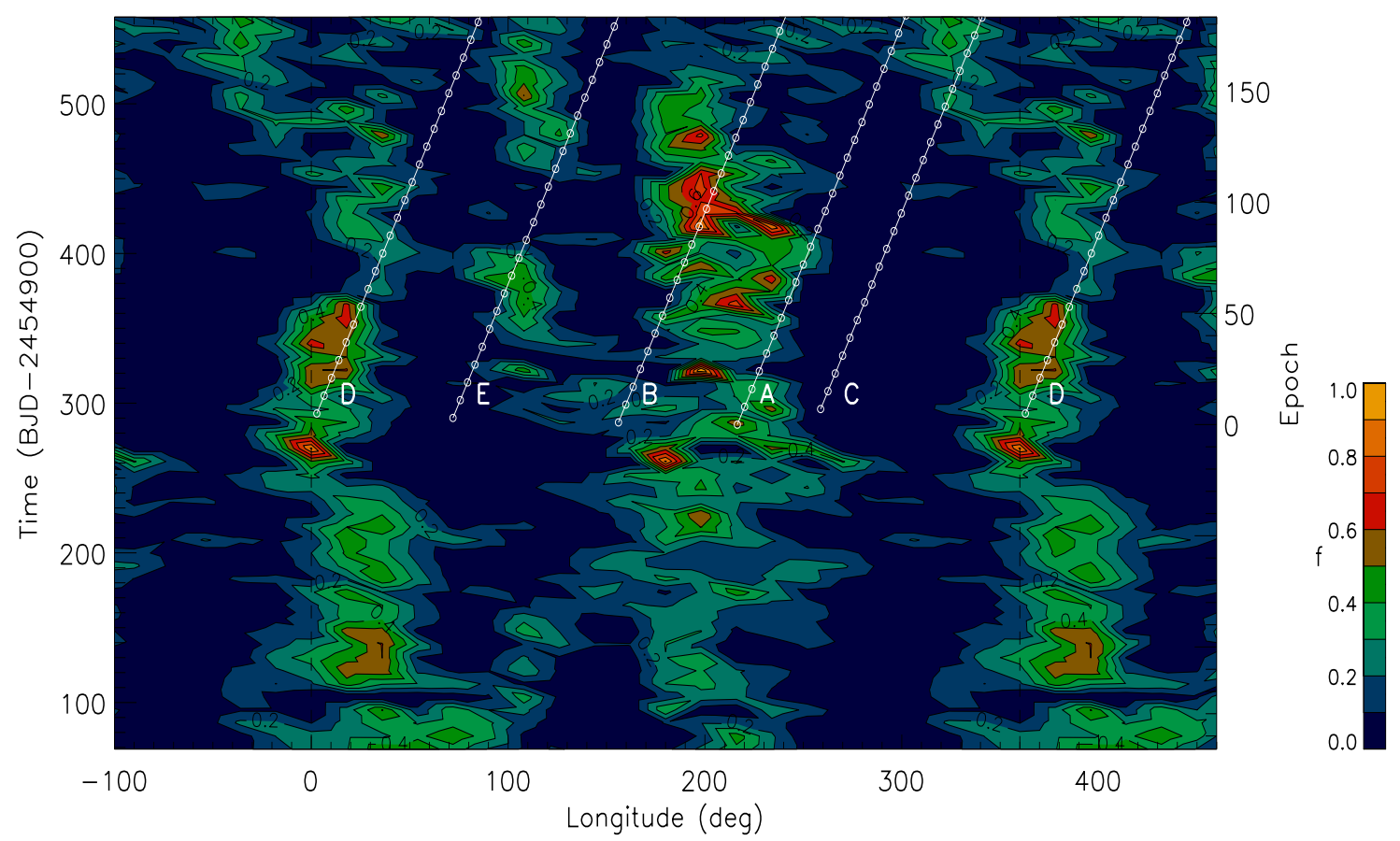

Fig. 4. Distribution of the spot-filling factor vs. longitude and time for $Q=1.6$. The values of the filling factor were normalized to their maximum $f_{\max }=0.01553$ with orange-yellow indicating the maximum and dark blue the minimum (see the colour scale on the right lower corner of the figure for the correspondence between the colour and the normalized filling factor). Note that the longitude scale is extended beyond $0^{\circ}$ and $360^{\circ}$ to help following the migration of the starspots. The tracks of the five spots occulted during the planetary transits after D11 are also reported. The open circles mark the time intervals of eight transits after which the same spots are detected again during the transits. The straight lines connecting the circles trace the migration of those spots in our reference frame; each line is labelled with the name of the corresponding spot, as indicated in Fig. 11 of D11.

on timescales shorter than three months. Moreover, the presence of gaps in the observations can introduce systematic errors in the measurement of the spotted area. This is the case of the gap beginning at BJD 2454997.982 for a duration of $5.0268 \mathrm{~d}$. Since the time interval adopted for our individual best fits is $\Delta t_{\mathrm{f}}=8.733 \mathrm{~d}$, this loss of data implies a systematically lower value of the total spotted area because the ME regularization removes spots at the longitudes not constrained by the observations. Fortunately, the other gaps in the photometric time series are much shorter than $\Delta t_{\mathrm{f}}$, thus no other value of the area is significantly affected. To show the distribution of the gaps, we plot line segments with a length equal to their duration at the level 0.04 in Fig. 5 considering all interruptions with a duration longer than $24 \mathrm{~h}$. In the analysis presented below, we discarded the area value at BJD 2455003.81 because it is affected by the gap that started at 2454997.982.

The Lomb-Scargle periodogram of the entire time series of the area values is plotted in Fig. 6 (solid line) together with the power level corresponding to a false-alarm probability of 0.01 as evaluated according to Horne \& Baliunas (1986). The main peak corresponds to a period of $47.1 \pm 4.5 \mathrm{~d}$ and has a false-alarm probability (FAP) of 2.0 percent. The value of the FAP was confirmed by performing an analysis of 50000 random Gaussian noise time series with the same sampling as our area data series. We also plot the periodogram of the time interval from BJD 2455230.869 to 2455457.929 (dashed line) because we see a regular oscillation of the total spotted area with a period of $\approx 48 \mathrm{~d}$ during that interval in Fig. 5. The main periodogram peak corresponds to a period of $48.2 \pm 9.0 \mathrm{~d}$ with an FAP of 0.44 percent.

The time variation of the frequency of the spotted area modulation is best represented by means of a wavelet amplitude. We plot in Fig. 7 the amplitude of the Morlet wavelet versus the period and the time (see, e.g., Hempelmann \& Donahue 1997, for details). The wavelet parameters are adjusted to give a time resolution of $\approx 100 \mathrm{~d}$ for a period of about $48 \mathrm{~d}$ and a relative period resolution of $\triangle P / P \approx 0.06$. We see that in the initial part of the dataset there is a periodicity of $\approx 30 \mathrm{~d}$ that corresponds to secondary peaks in the periodogram of the whole time series whose false-alarm probability is $>50$ percent (cf. Fig. 6 , where the frequency resolution is better than in the case of the wavelet). On the other hand, during the second half of the time series we see a clear periodicity of $\approx 50 \mathrm{~d}$, which corresponds to the significant peak in the periodogram. We conclude that the total spotted area of Kepler-17 showed an oscillation with a period of $47.1 \pm 4.5 \mathrm{~d}$. This behaviour is reminiscent of the short-term oscillations of the total sunspot area found close to the maxima of some of the 11-year solar cycles. They were called Rieger cycles because they were first detected in the periodicity of occurrence of large solar flares by Rieger et al. (1984). In the Sun, the periodicity is $\approx 160 \mathrm{~d}$ with small variations from one cycle to the other, although only some of the sunspot maxima show evidence of this short-term periodicity (Oliver et al. 1998; Krivova \& Solanki 2002; Zaqarashvili et al. 2010). A behaviour similar to that of Kepler-17 was found in CoRoT-2, a G7V star that showed a Rieger-type cycle in the variation of its spotted area with a period of $28.9 \pm 4.3 \mathrm{~d}$ (Lanza et al. 2009a).

\section{Discussion and conclusions}

The application of a spot model to reproduce the optical light curve of Kepler-17 shows that the spot pattern is almost stable for a timescale of $\approx 9 \mathrm{~d}$ because the residuals to our best fits have a standard deviation of $\sigma \approx 3.3 \times 10^{-4}$ in relative flux units 


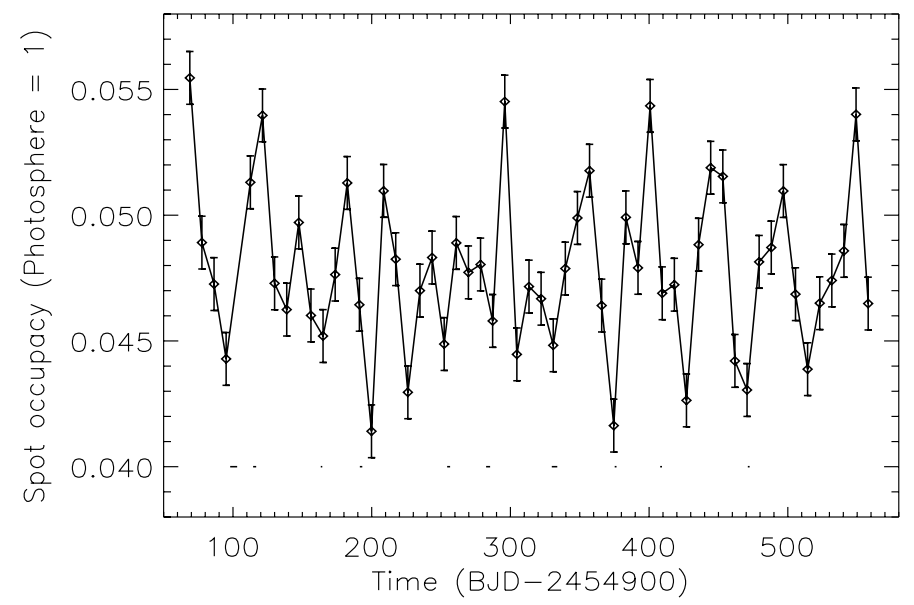

Fig. 5. Total spotted area as derived from the regularized ME models vs. time for $Q=1.6$. The lower horizontal ticks mark the gaps in the photometric time series longer than $24 \mathrm{~h}$.

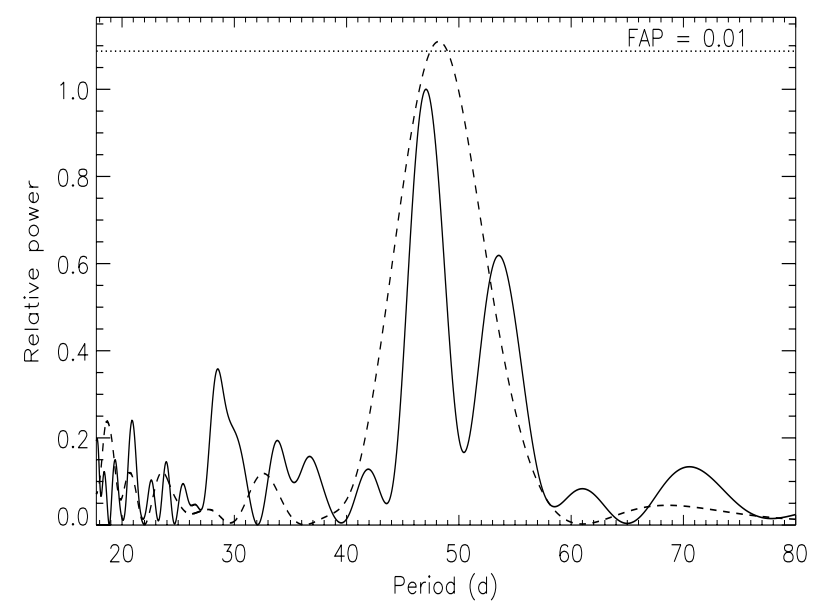

Fig. 6. Lomb-Scargle periodogram of the variation of the spotted area. The solid line gives the normalized power vs. the period for the whole time interval, while the dashed line gives the power for the time interval from BJD 2455230.8696 to BJD 2455457.9294 with the same normalization as adopted for the periodogram of the whole interval. The horizontal dotted line marks the 99 percent confidence level (FAP = $0.01)$.

that is only $\approx 50$ percent greater than the mean error attributed to the photometric measurements by the Kepler pipeline. Our facular-to-spotted area ratio $Q=1.6$ is significantly lower than the value $Q_{\odot}=9.0$ adopted for the modelling of the light curves of the Sun-as-a-star by Lanza et al. (2007). However, this lower value of $Q$ is typical of sun-like stars that are more active than the Sun (cf. Lanza et al. 2009a, 2010, 2011a,b) and suggests an increasing weight of the dark spots in the photometric variations as a star becomes more active, as indicated by the results of, e.g., Radick et al. (1998) and Lockwood et al. (2007).

Our models found several active longitudes that clustered on opposite hemispheres with a separation of $\approx 180^{\circ}$ for more than half the observation interval. This explains the two peaks found in the periodogram of the light curve by D11, one corresponding to the rotation period and the other to its first harmonic.

Bonomo et al. (2012) suggested an age younger than 1.8 Gyr, while D11 derived an age of $3.0 \pm 1.6$ Gyr for Kepler-17. As noted by B12, the age determined by means of standard gyrochronology is only $0.9 \pm 0.2 \mathrm{Gyr}$, while considering the effects of the close-in planet on the evolution of the stellar angular

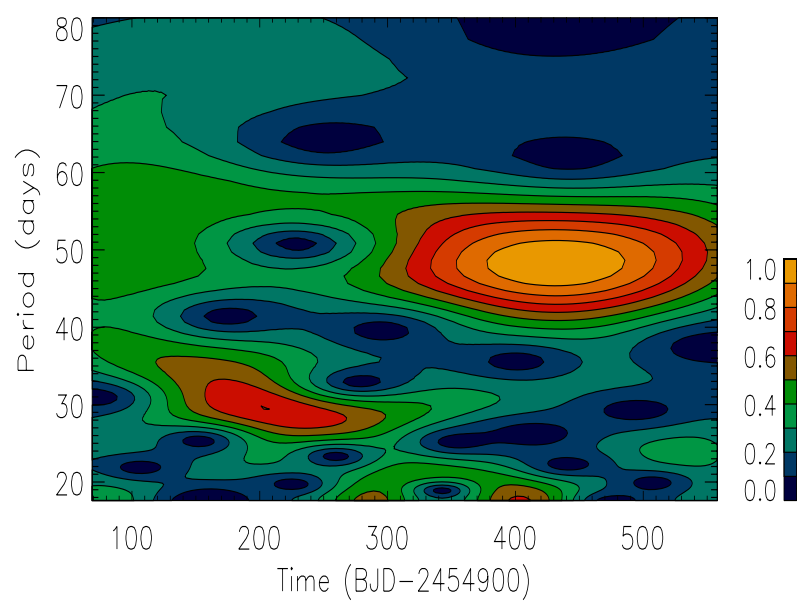

Fig. 7. Amplitude of the Morlet wavelet of the total spotted area variation vs. the period and the time. The amplitude was normalized to its maximum value. Different colours indicate different relative amplitudes from the maximum (yellow) to the minimum (dark blue) as indicated in the colour scale in the right lower corner.

momentum (Lanza 2010), an age of $1.7 \pm 0.3$ Gyr is estimated that seems more compatible with the age found from isochrone fitting.

Since the rotation period of the star is longer than the orbital period, tides remove angular momentum from the orbit to spin up the star and lead to orbital decay. The timescale for the engulfment of the planet can be estimated according to Ogilvie \& Lin (2007) as $\tau_{a} \simeq 0.048\left(Q_{\mathrm{s}}^{\prime} / 10^{6}\right) \mathrm{Gyr}$, where $Q_{\mathrm{s}}^{\prime}$ is the modified tidal quality factor of the star. This timescale is much shorter than the lifetime of the system on the main sequence if we adopt $Q_{\mathrm{s}}^{\prime} \approx 10^{6}$, i.e., the value derived from the observed circularization periods of close binary systems in open clusters of different ages. Together with the observations of several other stars with massive planets on very tight orbits, this suggests that $Q_{\mathrm{s}}^{\prime}$ should be much higher (i.e., the tidal dissipation much lower) in those star-planet systems than in close binary systems that consist of two main-sequence stars. The difference in the $Q_{\mathrm{s}}^{\prime}$ value could arise because the stellar rotation is far from being synchronized with the orbital motion of its planet. In this case, considering the dissipation of the tides inside the convection zone, Ogilvie \& Lin (2007) predicted $Q_{\mathrm{s}}^{\prime} \gtrsim 5 \times 10^{9}$, which implies an infall timescale longer than the system lifetime.

A lower limit on the value of $Q_{\mathrm{s}}^{\prime}$ can be set by an accurate timing of the transits over a time interval of a few decades because for $Q_{\mathrm{s}}^{\prime}=10^{6}$ we expect a variation of the orbital period of $\Delta P_{\text {orb }} / P_{\text {orb }} \approx 5 \times 10^{-8}$ in ten years. It produces a variation of $\approx 8 \mathrm{~s}$ in the epoch of the mid transit in 10 years. The accuracy reported by D11 is $\pm 2.4 \mathrm{~s}$ for their initial transit epoch, implying that a $Q_{\mathrm{s}}^{\prime}$ of about $10^{6}$ should give an orbital period acceleration detectable in a few years with a space-borne photometer. The recently approved extension of the Kepler mission till 2016 is therefore an interesting opportunity to perform such measurements. A model of the light perturbations that are caused by the spots occulted during the transits may possibly be used to improve the accuracy since D11 found small O-C timing oscillations with a period of approximately half the stellar rotation period, i.e., likely associated with starspots on opposite stellar hemispheres (cf. their Fig. 10).

We can estimate an approximate lower limit to the differential rotation of Kepler-17 finding $\Delta \Omega / \Omega \approx 0.10-0.16$ from the migration rates of the different trails of spots as seen in Fig. 4. Given the rapid evolution of the individual spots, this value is 
not only approximate, but depends critically on the way individual spot trails are identified. Therefore, we caution to regard this estimate as remarkably uncertain (cf. Sect. 5.2). For comparison, in $\mathrm{k}^{1}$ Ceti, a dwarf star with a spectral type G5V and a mean rotation period of $\approx 9 \mathrm{~d}$, Walker et al. (2007) estimated a relative differential rotation amplitude of $\approx 0.090 \pm 0.006$ by modelling the optical photometry obtained by the satellite MOST. A result similar to that of Kepler-17 was found by Croll et al. (2006) for $\epsilon$ Eridani, a K2V star with a rotation period of $\approx 11.4 \mathrm{~d}$, showing a relative amplitude of the differential rotation of $0.11 \pm 0.03$. On the other hand, the more rapidly rotating star CoRoT-2 $\left(P_{\text {rot }} \approx 4.52 \mathrm{~d}\right.$ ) shows very little surface differential rotation with a relative amplitude smaller than $\approx 1$ percent (Lanza et al. 2009a). In the young ( $\approx 50 \mathrm{Myr}) \mathrm{K} 2 \mathrm{~V}$ star KIC 8429280 , Frasca et al. (2011) found $\Delta \Omega / \Omega \approx 0.05$ while for the $\mathrm{G} 2 \mathrm{~V}$ stars KIC 7985370 and KIC 7765135, which have an age of $100-200$ Myr, Fröhlich et al. (2012) found $\Delta \Omega / \Omega \approx 0.07-0.08$. The differences can be attributed to the dependence of the differential rotation amplitude on the stellar rotation period and effective temperature as well as to the limited range of latitudes covered by starspots that can vary along the activity cycle. Moreover, the amplitude of the differential rotation could change along the activity cycle in rapidly rotating, highly active stars, such as the above mentioned Kepler targets that have $P_{\text {rot }}$ ranging from 1.2 and 2.8 d (cf. Frasca et al. 2011; Fröhlich et al. 2012). Therefore, individual results coming from spot modelling can be compared only in a statistical sense. Considering the general trend found by Barnes et al. (2005), the amplitude of the differential rotation estimated for Kepler-17 appears within the expected range for a star with its rotation period and effective temperature. Nevertheless, a more extended series of data is required to derive a firm conclusion on this point as well as on a possible influence of the planet on spot activity as conjectured by, e.g., Lanza (2008, 2011).

An interesting result of our analysis is the short-term spot cycle with a period of $47.1 \pm 4.5 \mathrm{~d}$, which is clearly detected in the second half of the data series. This phenomenon is reminiscent of the solar Rieger cycles because of its timescale and transient nature. The periodicity of the spotted area variations is close to 4 times the mean rotation period of the star, while in the Sun it is $\approx 6$ times and in CoRoT- 2 is $\approx 6.5$ times the rotation period. Those oscillations of the spotted area may be associated with hydromagnetic Rossby-type waves propagating in the upper part of the convection zone or at the interface between the radiative interior and the convection zone where the dynamo is probably located (Lou 2000; Zaqarashvili et al. 2010). Since only a few examples of stars displaying Rieger-type cycles are known (cf., Massi et al. 2005; Lanza et al. 2009a), the new results on Kepler17 are particularly interesting for a better understanding of this phenomenon in the framework of the solar-stellar connection.

As new Kepler data become available, it will be possible to refine the conclusions of the present study by investigating a longer time span. This is needed to assess the duration and the frequency of the Rieger-type oscillations in the total starspot area as well as to derive a definite conclusion on the possible impact of the planet on stellar activity.
Acknowledgements. The authors wish to thank an anonymous referee for several valuable comments, the NASA and the Kepler team for giving public access to the Kepler data. A. S. Bonomo gratefully acknowledges support through an INAF/HARPS-N fellowship. Active star research and exoplanetary studies at INAF-Osservatorio Astrofisico di Catania and Dipartimento di Fisica e Astronomia dell'Università degli Studi di Catania are funded by MIUR (Ministero dell'Istruzione, dell'Università e della Ricerca) and by Regione Siciliana, whose financial support is gratefully acknowledged. This research has made use of the ADS-CDS databases, operated at the CDS, Strasbourg, France.

\section{References}

Alonso, R., Auvergne, M., Baglin, A., et al. 2008, A\&A, 482, L21

Barnes, J. R., Collier Cameron, A., Donati, J.-F., et al. 2005, MNRAS, 357, L1

Bonomo, A. S., Hébrard, G., Santerne, A., et al. 2012, A\&A, 538, A96 (B12)

Borucki, W. J., Koch, D., Basri, G., et al. 2010, Science, 327, 977

Chapman, G. A., Cookson, A. M., \& Dobias, J. J. 1994, ApJ, 432, 403

Croll, B., Walker, G. A. H., Kuschnig, R., et al. 2006, ApJ, 648, 607

Désert, J.-M., Charbonneau, D., Demory, B.-O., et al. 2011, ApJS, 197,14 (D11)

Frasca, A., Fröhlich, H.-E., Bonanno, A., et al. 2011, A\&A, 532, A81

Fröhlich, H.-E., Küker, M., Hatzes, A. P., \& Strassmeier, K. G. 2009, A\&A, 506, 263

Fröhlich, H.-E., Frasca, A., Catanzaro, G., et al. 2012, A\&A, 543, A146

Hempelmann, A., \& Donahue, R. A. 1997, A\&A, 322, 835

Horne, J. H., \& Baliunas, S. L. 1986, ApJ, 302, 757

Huber, K. F., Czesla, S., Wolter, U., \& Schmitt, J. H. M. M. 2010, A\&A, 514, A39

Jenkins, J. M., Caldwell, D. A., Chandrasekaran, H., et al. 2010a, ApJ, 713, L120

Jenkins, J. M., Caldwell, D. A., Chandrasekaran, H., et al. 2010b, ApJ, 713, L87

Koch, D. G., Borucki, W. J., Basri, G., et al. 2010, ApJ, 713, L79

Krivova, N. A., \& Solanki, S. K. 2002, A\&A, 394, 701

Kurucz, R. L. 2000, http: //kurucz .harvard.edu/

Lampton, M., Margon, B., \& Bowyer, S. 1976, ApJ, 208, 177

Lanza, A. F. 2008, A\&A, 487, 1163

Lanza, A. F. 2010, A\&A, 512, A77

Lanza, A. F. 2011, Ap\&SS, 336, 303

Lanza, A. F., Catalano, S., Cutispoto, G., Pagano, I., \& Rodonò, M. 1998, A\&A, 332,541

Lanza, A. F., Rodonò, M., Pagano, I., Barge P., \& Llebaria, A. 2003, A\&A, 403, 1135

Lanza, A. F., Rodonò, M., \& Pagano, I. 2004, A\&A, 425, 707

Lanza, A. F., Bonomo, A. S., \& Rodonò, M. 2007, A\&A, 464, 741

Lanza, A. F., Pagano, I., Leto, G., et al. 2009a, A\&A, 493, 193

Lanza, A. F., Aigrain, S., Messina, S., et al. 2009b, A\&A, 506, 255

Lanza, A. F., Bonomo, A. S., Moutou, C., et al. 2010, A\&A, 520, A53

Lanza, A. F., Boisse, I., Bouchy, F., Bonomo, A. S., \& Moutou, C. 2011a, A\&A, 533, A44

Lanza, A. F., Bonomo, A. S., Pagano, I., et al. 2011b, A\&A, 525, A14

Lockwood, G. W., Skiff, B. A., Henry, G. W., et al. 2007, ApJS, 171, 260

Lou, Y.-Q. 2000, ApJ, 540, 1102

Massi, M., Neidhöfer, J., Carpentier, Y., \& Ros, E. 2005, A\&A, 435, L1

Mosser, B., Baudin, F., Lanza, A. F., et al. 2009, A\&A, 506, 245

Ogilvie, G. I., \& Lin, D. N. C. 2007, ApJ, 661, 1180

Oliver, R., Ballester, J. L., \& Baudin, F. 1998, Nature, 394, 552

Pagano, I., Lanza, A. F., Leto, G., et al. 2009, Earth Moon and Planets, 105, 373

Radick, R. R., Lockwood, G. W., Skiff, B. A., \& Baliunas, S. L. 1998, ApJS, 118,239

Rieger, E., Kanbach, G., Reppin, C., et al. 1984, Nature, 312, 623

Shkolnik, E., Bohlender, D. A., Walker, G. A. H., \& Collier Cameron, A. 2008, ApJ, 676, 628

Silva-Valio, A., \& Lanza, A. F. 2011, A\&A, 529, A36

Silva-Valio, A., Lanza, A. F., Alonso, R., \& Barge, P. 2010, A\&A, 510, A25

Walker, G. A. H., Croll, B., Kuschnig, R., et al. 2007, ApJ, 659, 1611

Zaqarashvili, T. V., Carbonell, M., Oliver, R., \& Ballester, J. L. 2010, ApJ, 709, 749 